\title{
ADAPTIVE CHANNEL ESTIMATION FOR UNDERWATER ACOUSTIC MIMO OFDM SYSTEMS
}

\author{
Milica Stojanovic \\ Northeastern University \\ Department of Electrical and Computer Engineering, 409 DA \\ Boston, MA 02115 \\ millitsa@ece.neu.edu
}

\begin{abstract}
Long acoustic multipath limits the applicability of MIMO OFDM channel estimation methods that require matrix inversion of size $M_{T} L$, where $M_{T}$ is the number of transmit elements and $L$ is the multipath spread measured in $1 / B$, the bandwidth inverse. To overcome this problem, sparse nature of the channel is exploited in an algorithm based on a compact signal representation that leads to two forms of adaptive implementation: one that requires matrix inversion but of a reduced size, and another that completely eliminates it. Channel estimation is coupled with phase tracking and prediction to enable decision-directed operation in the presence of nonuniform Doppler distortion, which in turn provides improved performance and reduced overhead. Performance is demonstrated on real data, recorded during a recent experiment in the coastal North Atlantic.
\end{abstract}

Index Terms - MIMO, OFDM, non-uniform Doppler, adaptive channel estimation, underwater acoustics.

\section{INTRODUCTION}

An underwater acoustic channel is characterized by a fundamental bandwidth limitation (propagation is best supported at low frequencies, e.g. $10 \mathrm{kHz}$ for distances on the order of $1 \mathrm{~km}$ ), extended multipath (tens of ms), and severe Doppler distortion caused by the inevitable motion and the low speed of sound $(1500 \mathrm{~m} / \mathrm{s}$ nominally). Although the operational bandwidth may be limited to only a few $\mathrm{kHz}$, an acoustic communication system is inherently (ultra) wideband since its bandwidth is not negligible with respect to the center frequency (on the contrary, the two can be comparable). This fact results in Doppler shifting that is not uniform across the signal bandwidth.

Orthogonal frequency division multiplexing (OFDM) has recently been considered as a low-complexity alternative to traditional single-carrier modulation for acoustic communications, with the focus on demonstrating its viability in the

This work was supported by the ONR grants N00014-07-1-0202 and N00014-07-1-0738. presence of non-uniform Doppler distortion. Two approaches have been pursued: one based on the classical principles of pilot-assisted, block-oriented detection [1], and another based on decision-directed, adaptive block processing $[2,3]$. The latter approach relies on frequency offset tracking and phase prediction to provide reliable symbol decisions, which in turn enable reduction in the pilot overhead, and can also lead to an improved performance.

To increase the bit rate over a limited acoustic bandwidth, we consider a multi-input multi-output (MIMO) spatial multiplexing system. We adopt the framework of decision-directed adaptive block processing, making use of the Doppler compensation principle [2] and focusing on channel estimation.

The problem of channel estimation in a MIMO OFDM system operating with $M_{T}$ transmit and $M_{R}$ receive elements is that of finding $M_{T} M_{R}$ frequency-domain channel coefficients for each of the $K$ carriers. ${ }^{1}$ The channel between the $t$-th transmitter and $r$-th receiver can also be described by an impulse response, requiring $L$ coefficients in the time domain. In a bandwidth-efficient acoustic system, $K$ can be (much) greater than $L$, making it advantageous to estimate the channel in the impulse response domain, converting later into the transfer function domain for data detection.

The received signal in each subband is related to $M_{T}$ transmitted data symbols, and, hence, a sufficient number of observations ( $M_{T} L$ subbands) have to contain known symbols. In block-oriented processing, these symbols must be known a-priori (pilots or null carriers) so that the channel can be estimated from the signals observed in the corresponding subbands. In contrast, block-adaptive processing utilizes symbol decisions, and channel estimation can benefit from signals received on all carriers. In either case, the solution to the channel estimation problem, be it of least squares (LS), minimum mean squared error (MMSE), or maximum a-posteriori probability (MAP) type, involves a matrix inverse of size $M_{T} L$. This fact has motivated the development of pilot allocation strategies and channel estimation algo-

\footnotetext{
${ }^{1}$ Similar problems are found in dual-polarization radio systems and multiwire channels [4].
} 
rithms whose goal is to avoid matrix inversion or reduce its complexity. Literature in the general area of (radio) communications abounds on this topic. Reduction in complexity has been sought through selection of significant impulse response coefficients which results in a reduced-size matrix inversion [5, 6]. The adaptive algorithm presented in Ref.[7] eliminates the need for matrix inversion by estimating each transmitter's response separately, having canceled the interference of other transmitter(s) using channel estimates from a previous block. This reference also provides optimal pilot sequences that simultaneously avoid matrix inversion and provide MMSE performance. The complexity of the algorithm can further be reduced by exploiting the correlation between adjacent carriers [8]. The idea of decomposing the received signal into individual transmitters' contributions has further been explored in Ref. [9], where the expectation-maximization (EM) principle is used to arrive at the LS channel estimates in an iterative manner. The same MIMO-SIM0 decomposition is utilized in a MAP channel estimator [10], which exploits low-rank approximation [11] to avoid matrix inversion. Channel estimation for MIMO sytems that aim for diversity gain through space-time coding is addressed in Ref.[12].

MIMO OFDM over acoustic channels has been demonstrated only recently $[13,14]$. Ref. [13] presents a classical block-oriented approach in which the problem of multiple channel estimation is decomposed into sequential estimation of individual channels by sending pilot tones from one transmitter at a time. This approach offers simplicity of implementation, but its overhead grows with the number of transmitters, eventually limiting the bit rate ( $L$ pilot tones and $\left(M_{T}-1\right) L$ null carriers must be reserved per transmitter). In an adaptive block processing approach [14], pilot tones are used only to initialize decision-directed channel estimation, which is performed in the transfer function domain, requiring parallel matrix inversions of size $M_{T}$ only. To provide sufficient number of observations, this approach assumes the channel transfer function to be identical between two (or more) adjacent carriers (and, if necessary, between adjacent blocks). By doing so, frequency correlation is exploited in a suboptimal, but computationally efficient manner.

To fully exploit the frequency correlation, we focus on LS channel estimation in the impulse response domain, seeking reduction in complexity (and improvement in performance) through channel sparsing and a block-adaptive method that eliminates the need for matrix inversion. Multiple channels are estimated simultaneously (rather than through successive interference cancellation) and the process is coupled with non-uniform frequency shift compensation.

The paper is organized as follows. After defining the system model in Sec.2, channel estimation is discussed in Sec.3. Sec. 4 is devoted to performance illustration using real data (256-carrier QPSK OFDM) recorded over a $2 \times 12$ shallow water channel in a $2.4 \mathrm{kHz}$ band centered at $11.2 \mathrm{kHz}$. Conclusions are summarized in Sec.5.

\section{SYSTEM MODEL}

The received signal after FFT demodulation is modeled as

$$
y_{k}^{r}(n)=\sum_{t=1}^{M_{T}} H_{k}^{t r}(n) d_{k}^{t}(n) e^{j \theta_{k}^{t}(n)}+z_{k}^{r}(n)
$$

where the indices $t, r, k, n$ refer to the transmitter, receiver, subband and time, respectively; $H$ refers to the channel, $z$ to the noise, and $d$ to the data symbols taken from an arbitry PSK/QAM alphabet. The phase shift is modeled as

$$
\theta_{k}^{t}(n)=\theta_{k}^{t}(n-1)+a^{t}(n) \cdot 2 \pi f_{k} T^{\prime}
$$

where $a^{t}(n)$ represents the residual Doppler factor (after initial resampling) corresponding to the $t$-th transmitter, ${ }^{2} f_{k}=$ $f_{0}+k \Delta f$ is the $k$ th carrier frequency, and $T^{\prime}=T+T_{g}$ is the time devoted to one OFDM block, which includes the signal of duration $T=1 / \Delta f$ and the multipath guard time $T_{g}$. Assuming that $a^{t}(n) f_{k}<<\Delta f, \forall t, k, n$, inter-carrier interference is treated as additional noise.

If we form the vectors

$$
\begin{aligned}
\mathbf{y}_{k}(n) & =\left[y_{k}^{1}(n) \ldots y_{k}^{M_{R}}(n)\right] \\
\mathbf{d}_{k}(n) & =\left[d_{k}^{1}(n) \ldots d_{k}^{M_{T}}(n)\right] \\
\mathbf{z}_{k}(n) & =\left[z_{k}^{1}(n) \ldots z_{k}^{M_{R}}(n)\right]
\end{aligned}
$$

and the matrices

$$
\begin{aligned}
\mathbf{H}_{k}(n) & =\left[H_{k}^{t r}(n)\right]_{t=1, \ldots M_{T} ; r=1, \ldots M_{R}} \\
\boldsymbol{\Theta}_{k}(n) & =\operatorname{diag}\left[e^{j \theta_{k}^{t}(n)}\right]_{t=1, \ldots M_{T}}
\end{aligned}
$$

we have that

$$
\mathbf{y}_{k}(n)=\mathbf{d}_{k}(n) \boldsymbol{\Theta}_{k}(n) \mathbf{H}_{k}(n)+\mathbf{z}_{k}(n)
$$

Given the channel matrix and the phases, the LS estimate of the data symbols transmitted on the $k$-th carrier is given by

$$
\hat{\mathbf{d}}_{k}(n)=\mathbf{y}_{k}(n) \mathbf{H}_{k}^{\prime}(n)\left[\mathbf{H}_{k}(n) \mathbf{H}_{k}^{\prime}(n)\right]^{-1} \boldsymbol{\Theta}_{k}^{*}(n)
$$

where the prime denotes conjugate transpose, and we are assuming that $M_{R} \geq M_{T}$. When the channels and the phases are not known, their estimates will be used instead of true values in the expression (9). Symbol decisions can then be made, e.g. by soft-decision decoding.

\section{CHANNEL ESTIMATION}

Let us define the discrete Fourier relationship

$$
\mathbf{H}_{k}(n)=\sum_{l=0}^{K-1} \mathbf{h}_{l}(n) e^{-j 2 \pi k l / K}
$$

\footnotetext{
${ }^{2}$ Experiments show that it suffices to use a single phase for multiple receivers [2, 3], but that different transmitters may require separate phases [14].
} 
where the coefficients $\mathbf{h}_{l}(n)=\left[h_{l}^{t r}(n)\right]_{t=1, \ldots M_{T} ; r=1, \ldots M_{R}}$ represent the MIMO channel in the impulse response domain. Note that fewer than $K$ impulse response coefficients may suffice to represent all of the $K$ transfer function coefficients. In particular, we define $J$ as the number of significant impulse response coefficients, and $L$ as their total contiguous span. The number of significant coefficients $J$ is normally not known a-priori, and neither are their positions $l_{j}, j=1, \ldots J$. However, their total extent $L$ is known from the expected multipath spread, which is a system design parameter in any OFDM system. Taking into account the fact that an underwater acoustic channel is rarely of minimum phase, we recognize that (10) can be written as

$$
\mathbf{H}_{k}(n)=\sum_{l=-A}^{L-1-A} \mathbf{h}_{l}(n) e^{-j 2 \pi k l / K}
$$

where $A \geq 0$, and it is understood that $\mathbf{h}_{l}(n)=\mathbf{h}_{K+l}(n)$ for negative values of $l$.

If we now form the matrices

$\mathbf{Y}(n)=\left[\begin{array}{l}\mathbf{y}_{0}(n) \\ \vdots \\ \mathbf{y}_{K-1}(n)\end{array}\right], \mathbf{D}_{\theta}(n)=\left[\begin{array}{l}\mathbf{d}_{0}(n) \boldsymbol{\Theta}_{0}(n) \\ \vdots \\ \mathbf{d}_{K-1}(n) \boldsymbol{\Theta}_{K-1}(n)\end{array}\right]$

and define $\boldsymbol{\Phi}=\operatorname{diag}\left[e^{-j 2 \pi k / K}\right]_{k=0, \ldots K-1}$, the received signals can be expressed in a simple form:

$$
\mathbf{Y}(n)=\sum_{l=-A}^{L-1-A} \boldsymbol{\Phi}^{l} \mathbf{D}_{\theta}(n) \mathbf{h}_{l}(n)+\mathbf{Z}(n)
$$

where the matrix $\mathbf{Z}(n)$ contains additive noise.

For purposes of channel estimation, it is convenient to express the received signal as

$$
\mathbf{Y}(n)=\boldsymbol{\Delta}(n) \mathbf{h}(n)+\mathbf{Z}(n)
$$

where

$$
\boldsymbol{\Delta}(n)=\left[\boldsymbol{\Phi}^{-A} \mathbf{D}_{\theta}(n) \ldots \boldsymbol{\Phi}^{L-1-A} \mathbf{D}_{\theta}(n)\right]
$$

and $\mathbf{h}(n)$ contains the corresponding terms $\mathbf{h}_{l}(n), l=$ $-A, \ldots L-1-A$. If all the data symbols are known, the LS channel estimate can be obtained as

$$
\hat{\mathbf{h}}(n)=\left[\boldsymbol{\Delta}^{\prime}(n) \boldsymbol{\Delta}(n)\right]^{-1} \boldsymbol{\Delta}^{\prime}(n) \mathbf{Y}(n)
$$

Note that in order for a solution to exist, the necessary condition is that $K \geq M_{T} L$. This condition can be interpreted in two ways: (1) for a given number of carriers $K$, at most $K / M_{T}$ channel coefficients can be estimated; and (2) for a given channel span $L$, at least $M_{T} L$ observations are needed. If fewer than $K$ observations are used, as would be the case in a block-oriented approach, then those rows of the matrices $\mathbf{Y}(n)$ and $\boldsymbol{\Delta}(n)$ that correspond to the pilot carriers will be isolated from the expression (13) to form a reduced set of $\left(P=M_{T} L\right)$ observations. The underlying data symbols ( $M_{T}$ per observation) have to be known. If all the data symbols can be known, as it is the case in a decision-directed approach, it is advantageous to utilize all $K$ observations instead of $M_{T} L$ only.

The knowledge of data symbols rests on the ability to accurately estimate the channel, and the assumption that the channel does not change much from one OFDM block to another. Channel estimates from the previous block can then be used to make tentative symbol decisions that will in turn be used to update the channel estimate. Note that pilot tones can also be used to aid decision-directed operation, but their number $P$ need not be constrained by the channel length.

The complexity of the problem can be reduced through channel sparsing. Namely, if only the $J$ significant channel entries are kept, the channel estimation problem (15) can be re-defined using

$$
\underline{\Delta}(n)=\left[\boldsymbol{\Phi}^{l_{1}} \mathbf{D}_{\theta}(n) \ldots \boldsymbol{\Phi}^{l_{J}} \mathbf{D}_{\theta}(n)\right]
$$

which will yield a corresponding channel estimate $\underline{\hat{\mathbf{h}}}(n)$ that now contains only the terms $\hat{\mathbf{h}}_{l_{j}}(n), j=1, \ldots J$. The matrix $\underline{\boldsymbol{\Delta}}^{\prime}(n) \underline{\boldsymbol{\Delta}}(n)$ is of size $M_{T} J$, and if $J$ is on the order of a few coefficients, ${ }^{3}$ the problem becomes manageable with several transmitters.

The set of significant coefficients, as we have used it, represents the union over all transmitters. This simplifies the notation, but it is not necessary. Nonetheless, when transmitters are co-located, it is reasonable to expect that their respective channels will indeed exhibit similar sparsing patterns.

To determine the positions of significant coefficients, fullsize estimation (15) can be performed initially. Since the initial estimate is made using known data symbols, the inverse $\left[\boldsymbol{\Delta}^{\prime}(0) \boldsymbol{\Delta}(0)\right]^{-1}$ can be pre-computed (the phase estimates are zero initially). Moreover, training symbols can be chosen so as to optimize the estimator and simultaneously trivialize the inversion [7].

The coefficients to be kept are determined as those whose magnitude exceeds some threshold. For example, a threshold can be set for each transmitter to $\gamma=20 \%$ of the magnitude of the strongest coefficient obtained for that transmitter. Some margin can also be used to set $J$ slightly greater than the value implied by initial channel estimation, to allow for the possibility of coefficient migration.

Once the significant coefficients have been initialized, channel estimation can switch to using the the reduced form (16) in a decision-directed manner of operation.

\footnotetext{
${ }^{3}$ Trials with experimental data [3] have shown that fewer than ten coefficients may suffice for a wideband $(19 \mathrm{kHz}-31 \mathrm{kHz})$ shallow water channel.
} 


\subsection{Adaptation}

An adaptive channel estimator can be obtained by filtering the instantaneous estimate, e.g. as

$$
\underline{\hat{\mathbf{h}}}(n)=\lambda \underline{\hat{\mathbf{h}}}(n-1)+(1-\lambda)\left[\underline{\Delta}^{\prime}(n) \underline{\Delta}(n)\right]^{-1} \underline{\Delta}^{\prime}(n) \mathbf{Y}(n)
$$

where $\lambda \in[0,1)$ accounts for the filter memory. This estimator still requires matrix inversion, and although its size has been reduced, further simplification may be desired. One way to address this problem is to replace $\underline{\Delta}^{\prime}(n) \underline{\underline{\Delta}}(n)$ by a stochastic approximation that would exploit the facts that the data symbols are uncorrelated, and rotated by different phases. We leave this exercise for later, and focus simply on inspecting the modeling equation:

$$
\mathbf{Y}(n)=\underline{\boldsymbol{\Delta}}(n) \underline{\mathbf{h}}(n)+\mathbf{Z}(n)
$$

This equation implies that $\underline{\Delta}(n)$ can be regarded as an input to a filter $\underline{\mathbf{h}}(n)$, whose output, in the presence of noise, is $\mathbf{Y}(n)$. Given that both the input and the output are known, the filter can be estimated in the least mean squares (LMS) manner as

$$
\underline{\hat{\mathbf{h}}}(n)=\underline{\hat{\mathbf{h}}}(n-1)+\mu \underline{\boldsymbol{\Delta}}^{\prime}(n)[\mathbf{Y}(n)-\underline{\boldsymbol{\Delta}}(n) \underline{\hat{\mathbf{h}}}(n-1)]
$$

where $\mu$ is the step size. Since the initial value $\underline{\hat{\mathbf{h}}}(0)$ is computed exactly using the known training symbols, slow convergence of the LMS algorithm is not an issue.

It may be worth noting that if we were to replace $\underline{\Delta}^{\prime}(n) \underline{\Delta}(n)$ by $K \mathbf{I}$, the expressions (17) and (19) would become identical with $\mu=(1-\lambda) / K$. This "replacement" corresponds to a stochastic approximation of $\underline{\underline{\Delta}}^{\prime}(n) \underline{\boldsymbol{\Delta}}(n)$ by its mean. Such an approximation is better justified when a greater number of observations are used for channel estimation (ideally all $K$ and not only the pilot channels).

After the channel estimate has been updated using either of the methods suggested, additional sparsing can be performed by setting to zero those elements of $\underline{\hat{\mathbf{h}}}(n)$ whose magnitude is below some threshold (not necessarily the same as the one used initially). This will result in $Q \leq J$ coefficients being kept on the average, and may further reduce the effect of channel estimation noise on data detection. There are two choices when it comes to updating: only those coefficients that have been kept can be passed on to the next block, or all can be passed, although only the selected ones will be used for data detection. The better choice depends on the channel dynamics, and is best made case-by-case.

Once the impulse response coefficients are available, the corresponding transfer function coefficients (11) can be computed for data detection (9). To do so, a set of FFTs can be applied according to the expression (10), taking care that the time-domain coefficients are appropriately arranged with zeros inserted into $K$-element vectors.

\subsection{Phase compensation}

Phase compensation in a MIMO system is based on estimating the Doppler factors $a^{t}(n)$ for all transmitters. This method is a straightforward extension of the SIMO case [2]. Assuming the existence of a previous estimate $\hat{a}^{t}(n-1)$ and the phase $\hat{\theta}_{k}^{t}(n-1)$, a prediction for the current block is made as

$$
\check{\theta}_{k}^{t}(n)=\hat{\theta}_{k}^{t}(n-1)+\hat{a}^{t}(n-1) \cdot 2 \pi f_{k} T^{\prime}, \forall k, t
$$

The existing channel estimate $\hat{\mathbf{H}}_{k}(n-1)$ is now used to form two types of symbol estimates according to the expression (9): $\check{\mathbf{d}}_{k}(n)$ is obtained using the predicted phase $\check{\theta}_{k}^{t}(n)$, and $\mathbf{d}_{k}(n)$ is obtained using the outdated phase $\hat{\theta}_{k}^{t}(n)$. The former is used to make tentative symbol decisions $\overline{\mathbf{d}}_{k}(n)$, as the latter may contain too large a phase offset. The underlying phase error is measured as

$$
\psi_{k}^{t}(n)=\left\langle\hat{d}_{k}^{t}(n) \bar{d}_{k}^{t *}(n)\right\rangle, \forall k, t
$$

and used to update the Doppler factors by averaging:

$$
\hat{a}^{t}(n)=\frac{1}{K} \sum_{k} \frac{\psi_{k}^{t}(n)}{2 \pi f_{k} T^{\prime}}
$$

These values are now used to update the phases,

$$
\hat{\theta}_{k}^{t}(n)=\hat{\theta}_{k}^{t}(n-1)+\hat{a}^{t}(n) \cdot 2 \pi f_{k} T^{\prime}, \forall k, t
$$

which are used to obtain the final symbol estimates and decisions. If the Doppler distortion can be modeled as equal for all transmitters (which is not always possible), the expression (22) will include additional averaging over the transmit elements.

\section{EXPERIMENTAL RESULTS}

An acoustic communications experiment known as "RACE" ( $\mathrm{R}$ stands for rescheduled) was conducted in March 2008 in the Narragansett Bay, RI, by the Woods Hole Oceanographic Institution (WHOI). The frequency range available for this experiment was $10 \mathrm{kHz}-12.4 \mathrm{kHz}$, and the signals were transmitted over a $1 \mathrm{~km}$ long, $9 \mathrm{~m}-14 \mathrm{~m}$ deep channel. Two transmitters, separated in the vertical by $60 \mathrm{~cm}$, were deployed $3 \mathrm{~m}$ above the sea floor. The receiver, positioned $2 \mathrm{~m}$ above the sea floor, consisted of a 12-element vertical array with a $10 \mathrm{~cm}$ element spacing.

Experimental signals were generated using $K=128$ and 256 carriers with QPSK modulation in each subband. The $\operatorname{BCH}(64,10)$ code was used so as to match the one currently implemented in the (single-carrier) WHOI acoustic modem. Each string of 10 bits was encoded into a 32-symbol codeword, and $K / 32$ such codewords were mapped onto the OFDM carriers keeping maximal separation between the symbols of the same codeword to gain frequency diversity. 
Confining the codewords to the same OFDM block does not exploit time diversity, but it enables instantaneous decoding for block-adaptive decision-directed operation.

Each transmission consisted of a frame ( $N$ OFDM blocks) carrying $2^{15}$ coded bits. The signal parameters are summarized in Table 1. QPSK bandwidth efficiency is given as the ratio of the bit rate to the bandwidth, $R_{b} / B=$ $2 M_{T} /(1+B T g / K)$. The effective bit rate is further reduced by coding; however, as the experimental results will demonstrate, a weaker code (as well as a shorter guard time) would have sufficed for the channel at hand.

\begin{tabular}{|l||c|c|c|c|}
\hline OFDM/QPSK & $K$ & $N$ & $\Delta f$ & $R_{b} / B$ \\
\hline$B=2.4 \mathrm{kHz}$ & 128 & 128 blocks & $18 \mathrm{~Hz}$ & $3 \mathrm{bps} / \mathrm{Hz}$ \\
\hline$T_{g}=16 \mathrm{~ms}$ & 256 & 64 blocks & $9 \mathrm{~Hz}$ & $3.5 \mathrm{bps} / \mathrm{Hz}$ \\
\hline
\end{tabular}

Table 1. Experimental signal parameters.

Fig.1 shows a snapshot of the channel obtained from an independent probe prior to OFDM signal transmission. A multipath spread of about $4 \mathrm{~ms}$ is observed, which translates into $L=10$ coefficients.

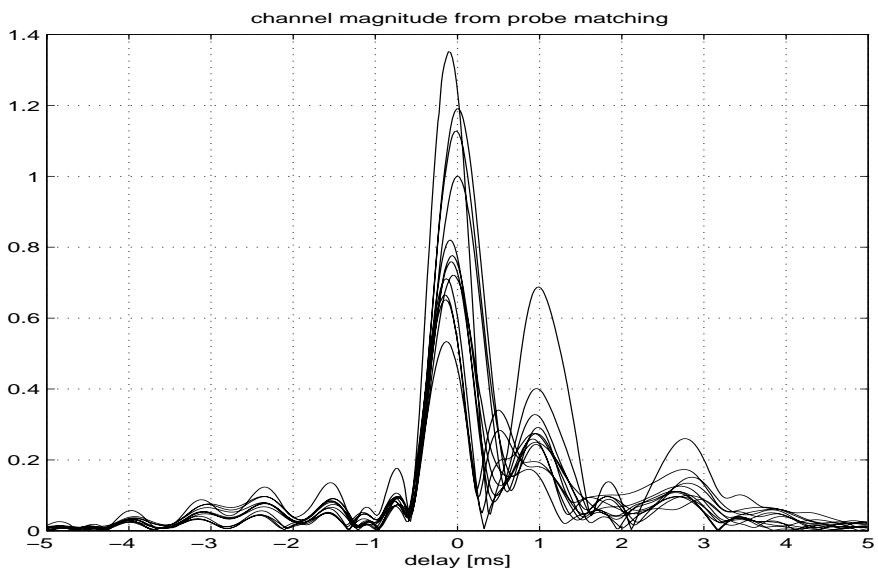

Fig. 1. Channel response estimates at 12 receivers are obtained by matched filtering to a 127 element PN sequence modulated onto an $11.2 \mathrm{kHz}$ carrier at $2.4 \mathrm{kbps}$.

Figs. 2 and 3 show the results of signal processing with $K=256$ (similar results were achieved with 128 carriers). OFDM was implemented with zero padding, and overlap adding [15] was performed prior to FFT demodulation to take into account $1 \mathrm{~ms}$ of the guard interval before, and $3 \mathrm{~ms}$ after an OFDM block. Shown in the figure are the phase estimates for several carriers, the channel responses for all receivers as seen at the end of the frame using algorithm (19), and the MSE in time (each value is an average over all carriers) and in frequency (each value is an average over all subbands). Various receiver parameters are listed in the figure, as well as the overall MSE (the average over all blocks and subbands) and the bit error rate (BER) in the frame.
The initial size of the channel estimator was set to $L=10$, and $A=1$ coefficient was reserved for the "anti-causal" multipath. A threshold $\gamma=0.2$ yielded $J=4$ significant coefficients. The first block was used to initialize the algorithm, and no pilots were used afterwards. Further sparsing resulted in $Q=2.1$ coefficients being kept on the average for the first transmitter's response and $Q=3.1$ for the second (the average is taken over time and over the receiving elements). Without sparsing, an MSE penalty of $1.8 \mathrm{~dB}$ was incurred.

Reducing the number of receivers from $M_{R}=12$ to 4 resuled in a $1.5 \mathrm{~dB}$ loss in the MSE, and another $1.5 \mathrm{~dB}$ was lost with $M_{R}=2$. However, no bit errors were observed in either case with the BCH code. Without coding, the BER was about 0.09 ( 0.05 if correct decisions are fed to the estimator). Choosing the step size $\mu$ on the order of $0.1 / K$ or less was found to be a good general rule.

The algorithm (17) yielded excellent performance as well, with low complexity $\left(M_{T} J=8\right)$. We emphasize, however, that the present experiment was conducted in a mild environment, using a bandwidth narrower than what could be available for the same transmission distance. A more complex environment could limit the applicability of the algorithm (17) to situations with manageable-size matrix inversion. In contrast, the algorithm (17) can easily be applied to a wide range of MIMO problems. Regardless of the adaptation method used, it must be kept in mind that data detection in a wideband acoustic system benefits from channel sparsing which eliminates the unnecessary estimation noise.

\section{CONCLUSIONS}

OFDM, which offers low-complexity post-FFT processing of multipath-distorted signals, was considered in a MIMO setting as a means of increasing the bit rate via spatial multiplexing over bandwidth-limited acoustic channels. To counteract the inevitable motion-induced Doppler distortion, nonuniform phase tracking was employed together with adaptive, decision-directed channel estimation. Discarding the insignificant channel coefficients provides improved performance on naturally sparse underwater channels, as well as the additional benefit of reducing the size of the channel estimation problem in a MIMO framework. An adaptive estimator of the LMS type was employed to further reduce the computational complexity by eliminating the need for matrix inversion. The algorithm was tested on experimental data, showing excellent performance at no pilot overhead. Future tests will target more challenging acoustic channels with wider system bandwidths.

When it comes to choosing the bandwidth, it may be interesting to note the subtle relationship between the number of significant coefficients $J$ and their total span $L$. Namely, the greater the bandwidth $B$, the greater will be the number of contiguous coefficients needed to span a given multipath spread $T_{m p}, L=\left\lceil B T_{m p}\right\rceil$. At the same time, the resolution 
$1 / B$ will improve, and the equivalent discrete-time channel coefficients will capture more of the physical propagation effect and less of delay dispersion caused by band-limiting at the transmitter. Hence, as $L$ increases above a certain limit, $J$ will reach a minimum. This minimum, which occurs as the bandwidth grows, will be equal to the number of physical propagation paths that significantly contribute to the received signals. Its exact value is determined by the system geometry, reflection and refraction properties, but in many situations of practical interest it will stay low enough to warrant MIMO processing of wideband acoustic signals.
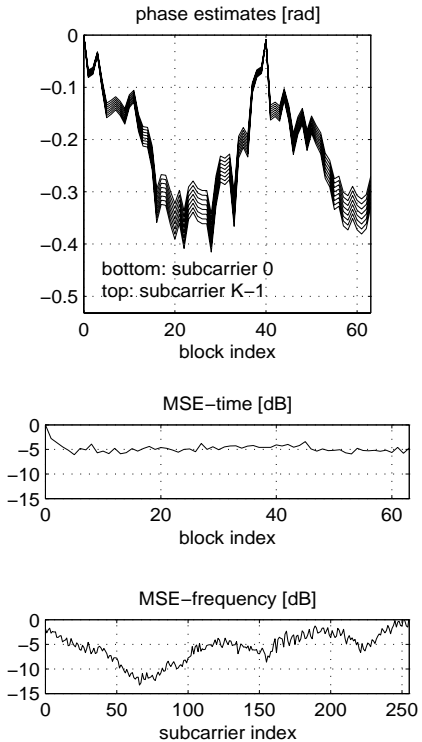

Fig. 2. Signal processing results for transmitter 1.
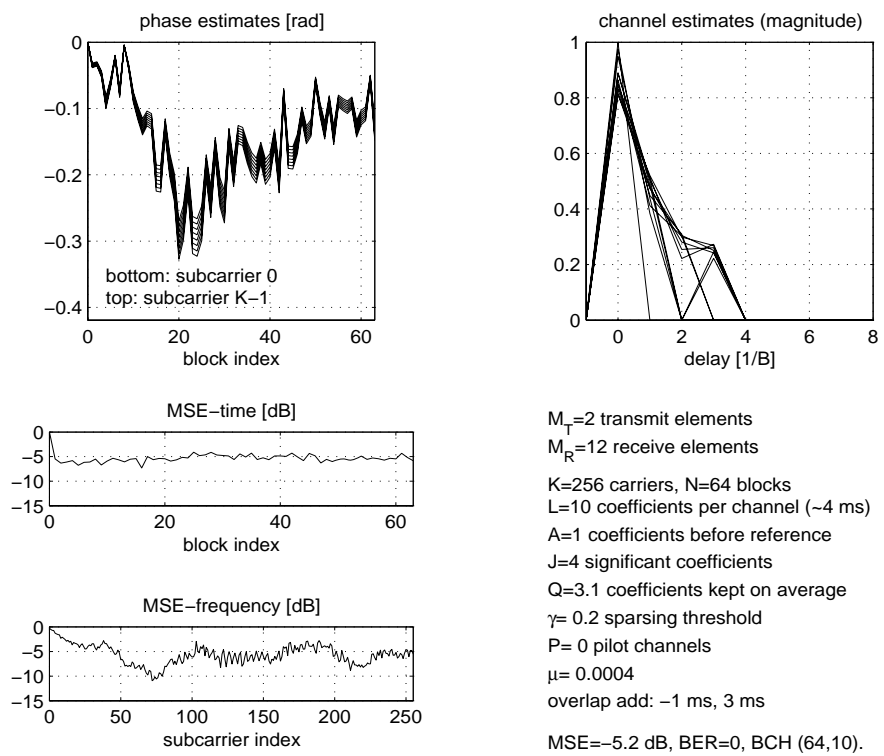

$M_{T}=2$ transmit elements $M_{R}=12$ receive elements $\mathrm{K}=256$ carriers, $\mathrm{N}=64$ blocks $\mathrm{L}=10$ coefficients per channel $(\sim 4 \mathrm{~ms})$ $\mathrm{A}=1$ coefficients before reference $\mathrm{J}=4$ significant coefficients $\mathrm{Q}=3.1$ coefficients kept on average $\gamma=0.2$ sparsing threshold $\mathrm{P}=0$ pilot channels $\mu=0.0004$

overlap add: $-1 \mathrm{~ms}, 3 \mathrm{~ms}$ $M S E=-5.2 d B, B E R=0, B C H(64,10)$.

Fig. 3. Signal processing results for transmitter 2 .

\section{REFERENCES}

[1] B.Li, S.Zhou, M.Stojanovic, L.Freitag and P.Willet, "Multicarrier communications over underwater acoustic channels with nonuniform Doppler shifts," IEEE Journal of Oceanic Engineering, to appear.

[2] M.Stojanovic, "Low complexity OFDM detector for underwater acoustic channels," in Proc. IEEE Oceans Conf., Sept. 2006.

[3] M.Stojanovic, "OFDM for underwater acoustic communications: adaptive synchronization and sparse channel estimation," in Proc. ICASSP, 2008.

[4] H.Nichols, A.Giordano and J.Proakis, "MLD and MSE algorithms for adaptive detection of digital signals in the presence of interchannel interference," IEEE Trans. Inform. Theory, vol.23, No.5, Sept. 1977, pp.563-575.

[5] Y.Li, N.Seshadri and S.Ariyavisitakul, "Channel estimation for OFDM systems with transmitter diversity in mobile wireless channels," IEEE J.Select. Areas Commun., vol.17, No.3, March 1999, pp.461-471.

[6] H.Minn and V.Bhargava, "An investigation into timedomain approach for OFDM channel estimation," IEEE Trans. Broadcast., vol.46, No.4., Dec. 2000, pp.240-248.

[7] Y.Li, "Simplified channel estimation for OFDM systems with multiple transmit antennas," IEEE Trans. Wireless Commun., vol.1, No.1, Jan. 2002, pp.67-75.

[8] K.Minn, D.Kim and V.Bhargava, "A reduced complexity channel estimation for OFDM systems with transmit diversity in mobile wireless channels," IEEE Trans. Commun., vol.50, No.5, May 2002, pp.799-807.

[9] Y.Xie and C.Georghiades, "Two EM-type channel estimation algorithms for OFDM with transmitter diversity," IEEE Trans. Commun., vol.51, No.1, Jan. 2003, pp.106115.

[10] J.Gao and H.Liu, "Low-complexity MAP channel estimation for mobile MIMO-OFDM systems," IEEE Trans. Wireless Commun., vol.7, No.3, March 2008, pp.774-780.

[11] O.Edfors, M.Sandell, J-J. ven de Beek, S.K.Wilson and P.O.Borjesson, "OFDM channel estimation by singular value decomposition," IEEE Trans. Commun., vol.47, No.7, July 1998, pp.931-933.

[12] Y.Li, J.Winters and N.Sollenberger, "MIMO-OFDM for wireless communications: signal detection with enhanced channel estimation," IEEE Trans. Commun., vol.50, No.9, Sept. 2002, pp.1471-1477.

[13] B.Li, S.Zhou, M.Stojanovic, L.Freitag and P.Willet, "MIMO OFDM over an underwater acoustic channel," in Proc. IEEE Oceans Conf., Oct. 2007.

[14] P.C.Ceballos and M.Stojanovic, "Adaptive MIMO detection of OFDM signals in an underwater acoustic channel," in Proc. IEEE Oceans Conf., 2008.

[15] B.Muquet, Z.Wang and G.Giannakis, "Cyclic prefix or zero padding for wireless multicarrier transmissions?" IEEE Trans. Commun., vol.50, No.12, Dec.2002, pp.21362148. 